\title{
Heterogeneity of Cortical Lesion Susceptibility Mapping in Multiple Sclerosis
}

\author{
(D) M. Castellaro, (D) Ragliozzi, (D) A. Palombit, (D). Pitteri, (DE. Silvestri, (D) V. Camera, (D) S. Montemezzi, (D) F.B. Pizzini, (D)A. Bertoldo, \\ (D) R. Reynolds, DS. Monaco, and (DM. Calabrese
}

\begin{abstract}
BACKGROUND AND PURPOSE: Quantitative susceptibility mapping has been used to characterize iron and myelin content in the deep gray matter of patients with multiple sclerosis. Our aim was to characterize the susceptibility mapping of cortical lesions in patients with MS and compare it with neuropathologic observations.
\end{abstract}

MATERIALS AND METHODS: The pattern of microglial activation was studied in postmortem brain tissues from 16 patients with secondary-progressive MS and 5 age-matched controls. Thirty-six patients with MS underwent $3 T$ MR imaging, including 3D double inversion recovery and 3D-echo-planar SWI.

RESULTS: Neuropathologic analysis revealed the presence of an intense band of microglia activation close to the pial membrane in subpial cortical lesions or to the WM border of leukocortical cortical lesions. The quantitative susceptibility mapping analysis revealed 131 cortical lesions classified as hyperintense; 33, as isointense; and 84, as hypointense. Quantitative susceptibility mapping hyperintensity edge found in the proximity of the pial surface or at the white matter/gray matter interface in some of the quantitative susceptibility mappinghyperintense cortical lesions accurately mirrors the microglia activation observed in the neuropathology analysis.

CONCLUSIONS: Cortical lesion susceptibility maps are highly heterogeneous, even at individual levels. Quantitative susceptibility mapping hyperintensity edge found in proximity to the pial surface might be due to the subpial gradient of microglial activation.

ABBREVIATIONS: $\mathrm{CL}$ = cortical lesion; DIR = double inversion recovery; EDSS = Expanded Disability Status Scale; $\mathrm{MHC}=$ major histocompatibility complex; MOG = myelin oligodendrocyte glycoprotein; $N A G M=$ normal-appearing gray matter; $n Q S M=Q S M$ value in the NAGM; $n Q S M_{\text {contra }}=$ median value of susceptibility from the reference tissue mask in the contralateral hemisphere; $\mathrm{nQSM}_{\text {surr }}=$ median value of susceptibility from the reference tissue mask surrounding the lesion; QSM = quantitative susceptibility mapping; RRMS = relapsing-remitting multiple sclerosis; SPMS = secondary-progressive multiple sclerosis

n

$n$ recent years, it has become increasingly clear that cortical and deep gray matter are not spared in multiple sclerosis. ${ }^{1,2}$ Several neuropathologic studies have consistently demonstrated that cortical gray matter lesions (CLs) are frequent in MS and that their accumulation strongly correlates with long-term disability measures. ${ }^{3}$ These observations have been confirmed and extended by

Received June 23, 2016; accepted after revision January 21, 2017.

From the Department of Information Engineering (M. Castellaro, A.P., E.S., A.B.), University of Padova, Padova, Italy; Neurology B (M. Castellaro, R.M., M.P., V.C., S.M., M. Calabrese), Department of Neurological, Biomedical and Movement Sciences, University of Verona, Verona, Italy; Division of Brain Sciences (R.M., R.R.), Faculty of Medicine, Imperial College London, Hammersmith Hospital, London, UK; and Neuroradiology and Radiology Units (S.M., F.B.P.), Department of Diagnostics and Pathology, Verona University Hospital, Verona, Italy.

M. Castellaro and R. Magliozzi contributed equally to the article.

Dr Calabrese had full access to all data in the study and takes responsibility for the integrity of the data and the accuracy of the data analysis. Dr Calabrese received honoraria for speaking from Sanofi-Aventis, Novartis Pharmaceuticals, Bayer Shering Pharma, Merck Serono, and Biogen Idec and funds for travel from Sanofi-Aventis, Genzyme, and Merck Serono. Dr Magliozzi was supported by the GR-20102313255 grant from Italian Ministry of Health. Drs Calabrese and Reynolds were supported by a Progressive Multiple Sclerosis Alliance grant (PA 0124). No other disclosures were reported. imaging studies showing that CLs correlate with both physical and cognitive disability. ${ }^{4,5}$ Unfortunately, despite these data, little is known about the pathogenetic mechanisms underlying CL development.

Neuropathologic studies have highlighted the lack of substantial focal immune infiltrates, complement deposition, and bloodbrain barrier damage in MS CLs ${ }^{6,7}$ and have suggested that meningeal inflammation and activated microglia may have a key role in GM damage. ${ }^{3,8}$ In particular, most of the examined CLs in postmortem brain samples exhibit a chronic inflammatory phenotype and rims of activated microglia close to the pial surface or the lesion edge. ${ }^{3,8,9}$

In a previous MR imaging/histopathologic combined study, ${ }^{10}$ hypointense rings on $\mathrm{T}_{2}^{*}$, representing activated microglia or

Please address correspondence to Massimiliano Calabrese, MD, Neurology B, Department of Neurological, Biomedical and Movement Sciences, University of Verona, Italy, Policlinico “G.B. Rossi” Borgo Roma, Piazzale L.A. Scuro, 10, 37134 Verona, Italy; e-mail: massimiliano.calabrese@univr.it

- Indicates open access to non-subscribers at www.ajnr.org

http://dx.doi.org/10.3174/ajnr.A5150

AJNR Am J Neuroradiol 38:1087-95 Jun 2017 www.ajnr.org 
macrophages, were observed at the edge of chronic active CLs. Similar rings have been reported in white matter lesions by using T2 ${ }^{*}$ phase imaging. ${ }^{11}$ These results, in line with those by Kooi et $\mathrm{al},{ }^{12}$ showed that some patients with MS had rims of activated microglia at the border of the CLs, whereas others did not. More recently, a study on ultra-high-field MR imaging on postmortem specimens of 2 patients with $\mathrm{MS}^{13}$ did not find rings of activated iron-laden microglia within CLs, and all CLs appeared darker in $\mathrm{R} 2 *$ images and brighter in magnitude images.

Magnetic susceptibility is a fundamental physical tissue property, which is known to reflect clinically relevant tissue characteristics, such as tissue iron content. During the last decades, phase imaging, ${ }^{14}$ SWI, ${ }^{15}$ and $\mathrm{T}^{*}{ }^{*}$ imaging $^{16}$ have been used to qualitatively assess magnetic susceptibility variations in cerebral tissue, including deep and cortical gray matter. ${ }^{17}$ Increased paramagnetic susceptibility-weighted filtered phase values were observed in the putamen in patients with clinically isolated syndrome compared with healthy controls. ${ }^{18}$ Thus, this finding suggests that susceptibility is sensitive to MS even in the early phase of the disease. In this context, quantitative susceptibility mapping (QSM), ${ }^{19}$ which overcomes several nonlocal restrictions of susceptibility-weighted and phase imaging, could shed some light on the pathologic process taking place in the cortical GM of patients with MS. A recent study has investigated MS, using QSM in the basal ganglia, ${ }^{20}$ showing that iron accumulation correlated with disease progression even in a patient with clinically isolated syndrome. Moreover, another group showed how WM MS lesions could be investigated longitudinally with QSM; the investigation could provide insight into the pathogenesis of those lesions. ${ }^{21}$ However, limited quantitative susceptibility data of cortical GM and, especially, of CLs are available. Indeed, only a recent study at 7T showed, ${ }^{22}$ in a restricted cohort of patients with MS, heterogeneity in CLs, with a predominant iron loss hypothesis.

In the present study, the characteristics of CLs were first determined with an analysis of microglial/macrophage activity in the postmortem MS brain, followed by the analysis of similar lesions in patients. 3D echo-planar imaging, which uses phase data to quantify local tissue susceptibility, was combined with a 3D double inversion recovery (DIR) at $3 \mathrm{~T}$ to characterize the in vivo susceptibility of CLs in patients with MS.

\section{MATERIALS AND METHODS \\ Neuropathologic Analysis}

This study was performed on postmortem brain tissues from 16 patients with secondary-progressive multiple sclerosis (SPMS) (mean age at death, $44.4 \pm 6.2$ years, Table 1 ; disease duration, $23.31 \pm 8.55$ years; time to wheelchair [from onset to Expanded Disability Status Scale \{EDSS\} 7], $41.21 \pm 7.83$ years; relapse rate in the first 2 years of the disease, $2.6 \pm 1.3$ ) selected for the presence of widespread cortical demyelination associated with meningeal inflammation and by more rapid and severe disease outcome associated with intense inflammatory activity among a larger group of 48 patients with SPMS previously extensively characterized for the presence and levels of inflammatory features and the extent of gray and white matter demyelination. ${ }^{23}$ Postmortem brain tissues from 8 controls, with no neurologic diseases, were also examined. In 3
Table 1: Neuropathologic details of postmortem brain tissue used in the study

\begin{tabular}{cccc}
\hline & $\begin{array}{c}\text { Sex/Age } \\
\text { at Death (yr) }\end{array}$ & $\begin{array}{c}\text { Postmortem } \\
\text { Delay (hr) }\end{array}$ & $\begin{array}{c}\text { Age at } \\
\text { Onset (yr) }\end{array}$ \\
\hline $\begin{array}{c}\text { SPMS cases } \\
\text { MS92 }\end{array}$ & $\mathrm{F} / 38$ & 26 & 21 \\
MS121 & $\mathrm{F} / 49$ & 24 & 36 \\
MS154 & $\mathrm{F} / 35$ & 12 & 23 \\
MS160 & $\mathrm{F} / 44$ & 18 & 29 \\
MS176 & $\mathrm{M} / 37$ & 12 & 10 \\
MS180 & $\mathrm{F} / 44$ & 9 & 26 \\
MS229 & $\mathrm{M} / 53$ & 13 & 37 \\
MS230 & $\mathrm{F} / 42$ & 31 & 22 \\
MS234 & $\mathrm{F} / 39$ & 15 & 23 \\
MS286 & $\mathrm{M} / 45$ & 7 & 29 \\
MS289 & $\mathrm{M} / 45$ & 9 & 27 \\
MS317 & $\mathrm{F} / 48$ & 21 & 18 \\
MS330 & $\mathrm{F} / 59$ & 24 & 19 \\
MS356 & $\mathrm{F} / 45$ & 10 & 28 \\
MS408 & $\mathrm{M} / 39$ & 21 & 29 \\
MS517 & $\mathrm{F} / 48$ & 12 & 23 \\
Control cases & & & \\
C14 & $\mathrm{F} / 64$ & 18 & \\
C25 & $\mathrm{M} / 35$ & 22 & \\
C28 & $\mathrm{F} / 60$ & 13 & \\
C30 & $\mathrm{M} / 75$ & 17 & \\
C36 & $\mathrm{M} / 68$ & 30 & \\
C41 & $\mathrm{M} / 51$ & 22 & \\
C48 & M/68 & 10 & \\
C54 & $\mathrm{M} / 66$ & 16 & \\
\hline
\end{tabular}

snap-frozen tissue blocks for each SPMS and control case, the presence and extent of demyelination and characterization of CL activity were determined by immunostaining with monoclonal antibodies for myelin oligodendrocyte glycoprotein (MOG) and major histocompatibility complex (MHC class II). ${ }^{3,24}$ CLs were classified, as previously described ${ }^{8}$ into leukocortical type I, intracortical type II, or subpial type III.

\section{In Vivo Study Population}

Thirty-six patients with $\mathrm{MS}^{25}$ having at least $1 \mathrm{MR}$ imagingvisible CL were studied (Table 2). Twenty-one had relapsingremitting MS (RRMS), and 15 had secondary-progressive MS. At study entry, most patients were under immunomodulatory therapy: Twelve were treated with interferon $\beta 1 \mathrm{a} / \mathrm{b} \beta 1 \mathrm{~b}$ or glatiramer acetate; 6 , with fingolimod; 4 , with natalizumab; 3 , with azathioprine; and 3 , with dimethyl fumarate; and 8 were untreated.

Each patient was assessed with the Expanded Disability Status Scale $^{26}$ and underwent $3 \mathrm{~T}$ MR imaging as described below. The study was approved by the University of Verona ethics committee, and informed consent was obtained from all patients.

\section{MR Imaging Acquisition and Analysis}

All patients were scanned with an Achieva 3T MR imaging scanner (Philips Healthcare, Best, the Netherlands). Isotropic 3D DIR $(1 \times 1 \times 1 \mathrm{~mm}, 10$ minutes 49 seconds $)$, 3D T1-MPRAGE $(1 \times$ $1 \times 1 \mathrm{~mm}, 5$ minutes 50 seconds $)$, and 3D EPI-SWI $(0.55 \times$ $0.55 \times 0.55 \mathrm{~mm}, 5$ minutes 51 seconds) images were acquired. Quantification of susceptibility maps was performed by using the recently introduced total generalized variation framework. ${ }^{27}$

After coregistration with 3D EPI, 3D DIR images were visually 
Table 2: Demographic, clinical, and QSM-related characteristics of the patient population

\begin{tabular}{lccc}
\hline & RRMS & SPMS & $\begin{array}{c}\text { Whole } \\
\text { Group }\end{array}$ \\
\hline No. & 21 & 15 & 36 \\
Age (yr) & $36.2 \pm 5.8$ & $49.5 \pm 9.2$ & $40.5 \pm 8.0$ \\
Disease duration (yr) & $9.7 \pm 6.3$ & $16.9 \pm 7.0$ & $12.7 \pm 7.5$ \\
Sex (F/M) & $16: 5$ & $9: 6$ & $25: 11$ \\
EDSS (mean) (range) & $2.0(1.0-5.5)$ & $5.0(4.0-7.0)$ & $3.0(1.0-7.0)$ \\
No. of intracortical lesions & & & \\
$\quad$ (mean) & & & \\
$\quad$ Total & $4.5 \pm 3.6$ & $2.1 \pm 2.6$ & $3.5 \pm 3.4$ \\
QSM-hyperintense & $2.8 \pm 2.3$ & $0.9 \pm 1.2$ & $2.0 \pm 2.1$ \\
QSM-isointense & $0.6 \pm 0.7$ & $0.1 \pm 0.4$ & $0.4 \pm 0.6$ \\
$\quad$ QSM-hypointense & $1.1 \pm 1.5$ & $0.9 \pm 1.8$ & $1.0 \pm 1.6$ \\
No. of leukocortical lesions & & & \\
$\quad$ (mean) & & & \\
Total & $2.6 \pm 3.1$ & $5.0 \pm 3.5$ & $3.6 \pm 3.5$ \\
QSM-hyperintense & $1.5 \pm 1.8$ & $1.9 \pm 1.5$ & $1.7 \pm 1.7$ \\
QSM-isointense & $0.5 \pm 0.9$ & $0.6 \pm 1.3$ & $0.5 \pm 1.1$ \\
$\quad$ QSM-hypointense & $0.5 \pm 0.9$ & $2.4 \pm 2.4$ & $1.3 \pm 1.9$ \\
No. of total lesions (mean) & & & \\
$\quad$ Total & $7.1 \pm 5.2$ & $7.1 \pm 5.0$ & $7.1 \pm 5.1$ \\
QSM-hyperintense & $4.2 \pm 3.4$ & $2.8 \pm 2.3$ & $3.6 \pm 3.0$ \\
QSM-isointense & $1.0 \pm 1.2$ & $0.7 \pm 1.3$ & $0.9 \pm 1.3$ \\
QSM-hypointense & $1.6 \pm 1.8$ & $3.3 \pm 3.7$ & $2.3 \pm 2.8$ \\
\hline
\end{tabular}

inspected and CLs were identified as intracortical or leukocortical lesions. Each CL was identified following the recent recommendations for CL scoring in patients with MS. ${ }^{28}$ All DIR images were assessed by consensus of experienced observers who were blinded to patient identity.

The appearance of CLs (identified with the DIR) on the QSM map was then evaluated. Each lesion was manually segmented on the DIR and then moved on the QSM. T1 was segmented to obtain a normal-appearing gray matter (NAGM) map. ${ }^{29,30}$ A threshold of $P>.9$ was used to ensure including mainly GM. The NAGM mask was then moved to the QSM space. The obtained GM mask was then used to segment a portion of the NAGM surrounding the lesion. Each CL mask was dilated (a circle of 7 pixels was used as the kernel) and was used to reduce the whole GM mask to the surrounding NAGM tissue. The surrounding mask was then refined by subtracting a dilated mask of the CL (circle of 3 pixels) to exclude the proximity of the lesion. We repeated the procedure, choosing a contralateral area of the brain as a reference (including only NAGM tissue). The median values of susceptibility from the reference tissue mask surrounding the lesion $\left(\mathrm{nQSM}_{\text {surr }}\right)$ and in the contralateral hemisphere $\left(\mathrm{nQSM}_{\text {contra }}\right)$ were subtracted from the QSM estimates within the CL. The distributions of both $\mathrm{nQSM}_{\text {surr }}$ and $\mathrm{nQSM}_{\text {contra }}$ were then tested with a $t$ test versus a zero-mean distribution. If both test results were statistically significant, the CL was classified as not isointense; otherwise, it was considered isointense. If the mean of both $\mathrm{nQSM}_{\text {surr }}$ and $\mathrm{nQSM}_{\text {contra }}$ was greater (lesser) than zero, the CL was classified as hyperintense (hypointense). If the mean value was discordant between the 2 references, the CL was discarded from the evaluation.

\section{Statistical Analysis}

Differences between RRMS and SPMS were assessed with ANOVA. The Pearson $\chi^{2}$ was applied to test the difference between the 2 patient groups in terms of categoric data (percentage of lesions). The Pearson correlation coefficient was used to assess the correlation between disease duration and lesion-counting metrics, whereas the Spearman coefficient was used to analyze the correlation with the EDSS. When we calculated correlations, a false discovery rate (with a false-positive rate of 0.05 ) correction technique was used to address multiple comparison correction issues. Each statistical test was considered significant with a level of .05 when not otherwise specified.

\section{RESULTS}

\section{Neuropathologic Analysis of Microglia Activity in CLs}

To evaluate the microglia/macrophage activation in the different types of CLs, we performed immunostaining for MHC class II and CD68 on tissue blocks from 16 SPMS cases. The activity of all 127 CLs identified in the examined SPMS cases was analyzed: the largest proportion $(45.0 \%)$ was chronic active, identified as cortical areas with MHC-II+ cells mainly localized at the lesion edge (Fig $1 A$ ), in agreement with previous studies. ${ }^{3,8}$ This type of CL was more abundant compared with both chronic inactive (25.9\%), with very low MHC II + cell density through the entire lesion, and active $(29.1 \%)$ CLs, characterized by numerous MHC-II+ lesions in the lesion core and borders (Fig 1A).

Examination of the inflammatory activity of large CLs (Fig 1B) showed that MHC class II immunostaining was mainly restricted to activated microglia with ramified morphology, with a higher density in the most external cortical layers close to the pial membrane in subpial type III CLs (Fig 1C) or close to the WM portion in type I CLs (Fig 1D), either in actively demyelinating or chronic active lesions. In chronic inactive lesions, a lower density of MHC-II+ cells was present, scattered in all demyelinated areas (Fig $1 E,-F$ ). Furthermore, for each of the examined patients with MS, all the different types of CL activity were found. Large CLs (Fig $1 B$ ) sometimes contained, simultaneously, rims of activated microglia either close to the pial surface (Fig 1C) or toward the WM lesion border (Fig 1D).

\section{Imaging Data}

Two hundred fifty-five CLs were identified in the living population. On the basis of the DIR sequence, 126 CLs were pure intracortical and 129 CLs were leukocortical; on the basis of QSM, 131 were hyperintense, 33 were isointense, and 84 were hypointense (Fig 2). Seven CLs were discarded from the analysis because discordant results of the $t$ test between $\mathrm{nQSM}_{\text {surr }}$ and nQSM $\mathrm{Bntra}_{\text {con }}$ were found. Among the 36 patients with MS, 32 showed at least 1 QSM-hyperintense CL, 16 showed at least 1 QSM-isointense CL, and 23 showed at least 1 QSM-hypointense CL. Twenty-six patients (72.22\%) showed at least 2 QSM subtypes of CLs, and 11 patients showed all QSM subtypes of CLs at the same time. Table 2 shows the differences between intracortical and mixed GM/WM lesions.

The number of hyperintense CLs was higher in RRMS (mean, $4.1 \pm 1.4$; range, 0-14) compared with SPMS (mean, $2.2 \pm 1.5$; range, $0-5$ ), while the number of hypointense CLs was higher in patients with SPMS (mean, $0.8 \pm 1.1$; range, $0-4$ in RRMS; mean, $3.5 \pm 3.5$; range, $0-11$ in SPMS). Indeed, in RRMS, $61.4 \%$ of CLs were hyperintense and $23.4 \%$ were hypointense, whereas in SPMS, $40.7 \%$ of CLs were hyperintense and $48.5 \%$ were hypointense. 

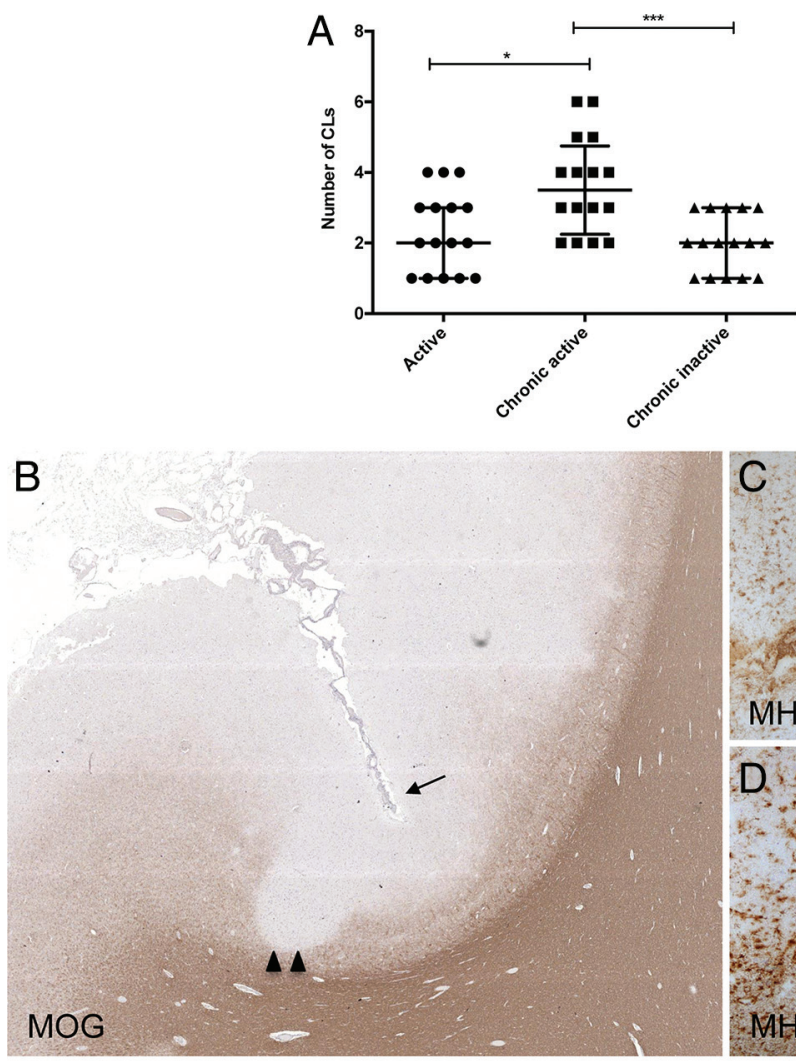

$\mathrm{E}$
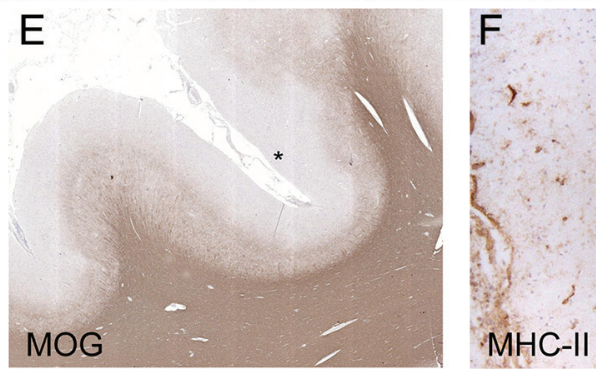

FIG 1. Neuropathologic assessment of cortical lesion activity. The number of active, chronic active, and chronic inactive GM lesions was evaluated with MHC class II immunostaining in 3 snap-frozen tissue blocks $(A)$ for each of the examined 16 postmortem MS cases. A significant increased level of chronic active $C L$ has been found compared with both active and chronic inactive CL. MOG immunostaining (B) shows an extensive chronic active subpial type III GM lesion, also reaching the WM (arrowheads). Combined MHC class II immunostaining of serial sections ( $C$ and $D$ ) demonstrates the high density of activated MHC-II + cells close to the pial surface of the lesion (arrow in $B$, higher magnification in $C$ ) and toward the WM edge of the lesion (arrowheads in $B$, higher magnification in $D$ ). In a chronic inactive subpial CL $(D)$, minimal density of $\mathrm{MHC}-\mathrm{II}+$ cells $(E)$ has been found. In extensive chronic inactive lesions shown by MOG immunostaining loss (E), the lower density of MHC-II+ cells was present and scattered in all the demyelinated area, also close to the pial surface (asterisk in $E$, and higher magnification in $F)$. Original magnifications $\times 25(B$ and $E), \times 200(C, D$, and $F)$.

\section{Neuropathology/Imaging Comparison}

The QSM hyperintensity edge found in proximity to the pial surface in some examined QSM-hyperintense CLs mirrored the intense microglia activation found close by the pial surface in most subpial CLs observed in postmortem SPMS cases. Indeed, the intense band of microglia activation frequently found at the depth of cerebral sulci of active CLs (Fig $3 A, B$, -E, blue arrowheads), with a characteristic gradient of increased microglia density in the external cortical layers (I-III), decreasing toward the most inner cortical layers (Fig 3E), resembled the hyperintense QSM signal seen in Fig 3J (blue arrowheads). Similar intense MHC-II immunostaining was also observed along the surface of the cerebral sulci (Fig 3A, - $C$ yellow arrowheads), especially in regions of active demyelinating subpial GM, comparable with the QSM hyperintensity edge found in proximity to the pial surface within cerebral sulci (Fig 3J, yellow arrowheads). Also, the QSM hyperintensity edge found at the WM/GM border seems to resemble the microglia/macrophage activation detected at the WM/GM interface in leukocortical lesions (Fig 3H). Most interesting, a marked difference in MHC-II+ density and morphology has been observed in WM and GM (Fig 3H) portions of type I CLs, suggesting functional differences in the resident microglia cells in these areas and a different ratio of microglia/monocytederived macrophages in the presence of a higher myelin density.

\section{DISCUSSION}

In this preliminary study, we combined neuropathologic analysis of GM lesion

A moderate inverse correlation was observed between hyperintense intracortical CLs and disease duration $(r=-0.48)$, while no correlation was observed between disease duration and hypointense or isointense CLs.

The total number of CLs correlated with EDSS $(r=0.51)$. In patients with RRMS, the correlation between both total QSMhyperintense CLs and intracortical hyperintense CLs with EDSS was marked ( $r=0.63$ and $0.71, P<.05)$, while no correlation was observed between EDSS and QSM-hypointense or isointense CLs. Moreover, no correlation was found among hyperintense, hypointense, or isointense CLs and EDSS in patients with SPMS. activity and a new imaging approach with 3D DIR and QSM, with the aim of better characterizing CLs in patients with RRMS and SPMS. Our results suggest that combined 3D DIR and QSM may reveal the area of intense microglia/macrophage activity and density in CLs as also detected by neuropathologic assessment. Furthermore, our data show that CLs are highly heterogeneous as revealed by their susceptibility maps. This heterogeneity was present across, and in some cases within, the lesions. These data are partially in contrast to a recent MR imaging study performed at $7 \mathrm{~T}$ showing negative relative susceptibility values in 25 of the 27 CLs. $^{22}$ However, the low number of patients and CLs analyzed might account for this discordance. Moreover, it is likely that the 


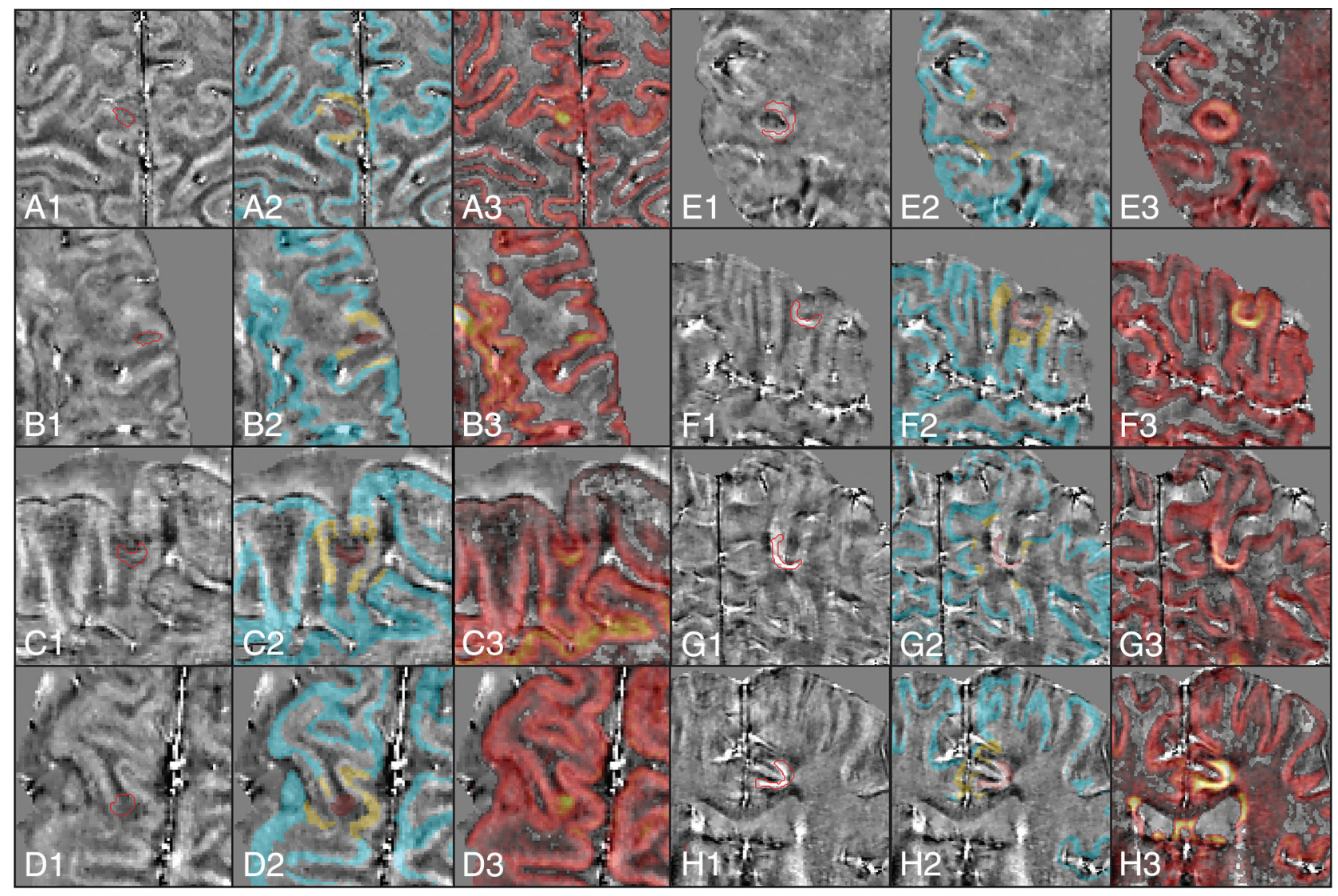

FIG 2. Illustration of examples of cortical lesion appearance on the quantitative susceptibility map obtained with the total generalized variation algorithm from the 3D EPI susceptibility-weighted scan. Each panel denoted by a letter is divided in 3 subpanels: 1) QSM with contoured CL (red line); 2) QSM with a superimposed CL (red), the NAGM reference tissue used in the CL classification (yellow), and NAGM obtained from the segmentation of the 3D T1 MPRAGE (cyan); 3) QSM with superimposed the 3D double inversion recovery sequence, where the CL detection and segmentation was performed. $A-D$, Hypointense lesions. $E-H$, Hyperintense lesions. The classification of $C L s$ was performed with a 2-sided $t$ test between QSM estimates in the lesion (with subtracted the median value of the reference QSM value in the NAGM) and a zero mean Gaussian distribution. When the $t$ test was significant and the mean of nQSM was greater (lesser) than zero, the lesion was classified as hyperintense (hypointense).

susceptibility of CLs changes with time in relation to their activity as previously shown for WM lesions. Therefore, the low activity of the patients with MS and of the number of CLs detected at the time of the MR imaging might also help explain this contradiction. ${ }^{31-33}$

The high variability of CLs in inflammatory activity in patients with MS has been already described by several neuropathologic studies mainly based on SPMS patients with a relatively long disease duration ${ }^{3,8,12,34}$ or on material from biopsies of patients with early MS with tumefactive lesions. ${ }^{9}$ Despite these neuropathologic observations, there are no available data in vivo that characterize the inflammatory activity of CLs in the early phase of the disease. In a previous MR imaging study, we observed that a small subgroup of CLs was significantly hyperperfused; this finding suggested the presence of an active inflammatory process within cortical GM. ${ }^{35}$

Although the number of patients with SPMS was quite low for drawing definitive conclusions, QSM-hyperintense CLs appeared to be more frequent in the RRMS group, while QSM-hypointense lesions were more frequent in SPMS. This result, along with the moderate inverse correlation between the number of QSM-hyperintense CLs and disease duration, might suggest that QSM-hyperintense lesions characterize the more inflammatory and acute phases of the disease, while QSM-hypointense CLs characterize the chronic (less inflammatory) disease stage. Nevertheless, the presence of each type of QSM-visible CL in both RRMS and SPMS suggests that the heterogeneity of GM lesion types persists during the entire disease course. Of course, we are aware that the DIR sequence detects only a reduced portion of CLs $s^{36}$ and that the use of other sequences such as phasesensitive inversion recovery might help improve their detection. ${ }^{37}$ However, recent comparative histologic/MR imaging studies have demonstrated that the "tip of the iceberg" detected by MR imaging and its "bulk" differ only in size and that the number of detectable CLs correlates with their overall number and with the overall percentage of cortical demyelination. ${ }^{36}$ The ability of QSM in detecting CLs itself has not been tested; however, we do not advise acquiring only the SWI sequence. Using the DIR or even the phase-sensitive inversion recovery sequence is recommended as a guide for detecting CLs.

Although the factors contributing to the susceptibility in the cortex are not fully established, ${ }^{38}$ initial studies of healthy brains both in vivo and postmortem have suggested that myelin and both heme and nonheme iron have dominant effects in conditioning the susceptibility map. ${ }^{39,40}$ In contrast to $\mathrm{R} 2^{\star}$, which increases proportionally to the concentration of both iron ${ }^{41}$ and myelin, ${ }^{42}$ the 2 substances have opposing effects on the magnetic 

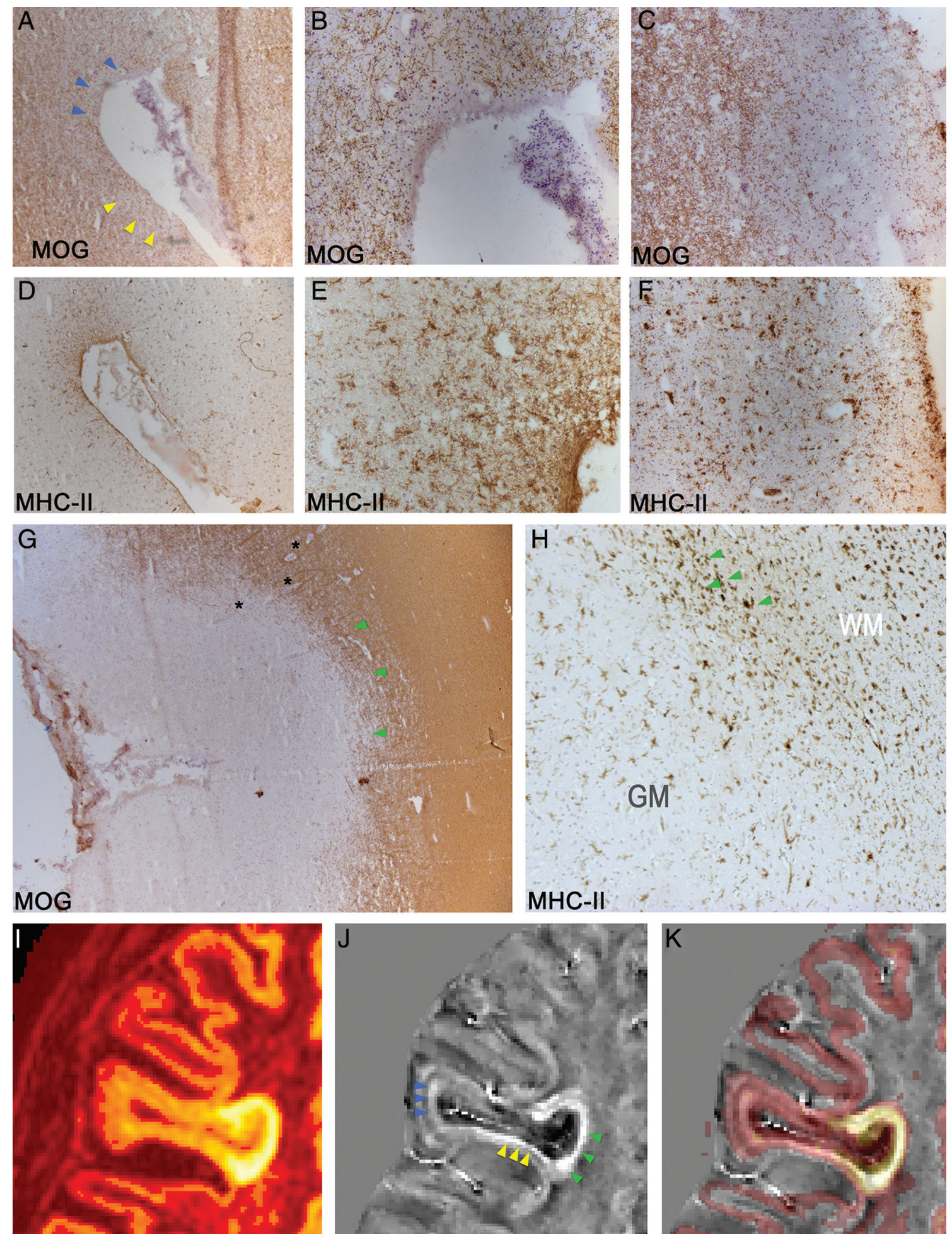

FIG 3. Combined neuropathologic and MR imaging characterization of lesion activity in CLs. $A-H$, Immunohistochemistry staining of MOG ( $A-C$ and $G)$ and MHC class II (D-F and $H)$ in subpial $(A-F)$ and leukocortical $(G$ and $H) C L s$ in postmortem MS brains. $I-K$, MR images of heterogeneous CLs in patients with MS in vivo; I, 3D double inversion recovery.J, Quantitative susceptibility mapping calculated with the total generalized variation algorithm. $K$, DIR superimposed on the QSM map. MOG and MHC-II immunostaining on serial sections show ongoing subpial demyelination (blue arrowheads in $A$, higher magnification in $B$ ) and intense pick of $M H C-I I+$ glia activation $(D$, higher magnification in $E$ ) at the depth of a cerebral sulcus, resembling the QSM hyperintense signal (blue arrowheads in /) in similar regions. Concurrent complete subpial demyelination (yellow arrowheads in $A$, higher magnification in $C$ ) and intense peak of MHC-II+ glia activation $(F)$ were also detected along the pial surface of the same cerebral sulcus (yellow arrowheads in A), respectively, resembling similar QSM hyperintense signal (yellow arrowheads in ). In type I leukocortical lesions, shown by MOG immunostaining $(G)$, high occurrence and density of MHC-II+ activated microglia and macrophages $(H)$ were observed at the GM/WM interface $(g r e e n$ arrowheads in $G$ and $H$ ). Higher magnification of the GM/WM interface (green arrowheads in $H$ ) reveals higher density of MHC-II+ cells mainly in proximity to inflammatory infiltrates (asterisks in $G$ ) within the WM border of the lesions, possibly corresponding to the frequent QSM hyperintense signal shown by green arrowheads in J. Original magnifications $\times 100(A, D$, and $G), \times 200(B, C, E, F$, and $H)$. 
susceptibility maps ${ }^{43,44}$ because the proteins and lipids associated with myelin render its susceptibility diamagnetic. ${ }^{45,46}$

Our neuropathologic data, in line with previous studies, ${ }^{3,8,47}$ confirmed that a significant proportion of type III and type I chronic active CLs are associated with a gradient of increased microglia activation in the most external cortical layer, close to the pial surface (in subpial type III CLs; Fig 3, yellow arrows) or to the WM interface (in leukocortical type I CLs; Fig 3, blue arrows). This finding was also observed in a recent neuropathologic study ${ }^{12}$ showing that in a subset of patients with MS with CLs, part of the CLs were characterized by a rim of activated microglia at their border. The examined postmortem SPMS cohort included MS cases that, at the time of death, had differences in both disease duration and clinical disability compared with the in vivo SPMS cases. These differences may explain the increased activity of the CLs detected in the postmortem SPMS cases.

Although our pathologic and MR imaging analyses are derived from 2 different cohorts of patients and therefore a direct comparison is only theoretic, on the basis of the similarity of the neuropathlogic and MR images, we hypothesize that the QSM-hyperintensity could be due to activated microglia/macrophages that phagocytose nonheme iron-rich cellular debris. As suggested by previous pathologic and imaging studies, ${ }^{3,10,48,49}$ the presence of a hyperintense rim in part of the detected CLs could indicate the presence of iron accumulation in microglia/macrophages at the subpial edge (in type III CLs) or at the WM/GM interface. If this is the case, the sensitivity of QSM in detecting activated microglia might explain some of the subpial hyperintensities observed with this sequence and not with the DIR sequence, which is usually not sensitive enough for subpial lesions (Fig 2). A recent study ${ }^{50}$ on a limited number of patients showed the capability of QSM to better discriminate intracortical and leukocortical lesions, thus suggesting that QSM could be useful in predicting and detecting early modifications of normal-appearing tissues. ${ }^{51}$ Nevertheless, further studies that combine postmortem MR imaging and neuropathologic analysis on the same cohort are currently in progress.

The QSM hyperintense signal frequently observed in proximity to, or within, the WM portion of the detected leukocortical lesions might resemble the similar increased density of MHC-II+ microglia found in the WM portion of the type I CLs. This resemblance might indicate the expanding inflammatory rim involved in the neuropathogenesis of type I CLs.

At least 2 alternative hypotheses might explain the presence of a hyperintense QSM signal: The hyperintense QSM signal could be generated by the increased iron release from intracellular deposits to extracellular spaces or by the iron leakage following blood-brain barrier damage in the acute phases of the disease. However, it is not usual within the cortical GM to have a BBB breakdown. ${ }^{52}$ The second hypothesis suggests that oligodendrocytes constitute an important source of iron, and changes in iron signal could be associated with a loss of oligodendrocytes and reduction of nonheme iron within oligodendrocytes and myelin in MS plaques and periplaque areas. ${ }^{52}$ Therefore, the detected QSM-hypointensity could be due to a decrease of nonheme tissue iron, which characterizes those inactive GM lesions without activated microglia and is more frequent in patients with a long disease duration. ${ }^{53}$

Several observations have suggested that an abnormal deposi- tion of iron might also play a significant role in the pathophysiology of GM damage in patients with $\mathrm{MS}^{54}$ as also is shown for many age-related degenerative disorders. ${ }^{55}$ While confirming the well-known relationship between CLs and disability, ${ }^{56}$ our data have extended these results, showing a significant correlation between QSM-hyperintense CLs (more than the hypointense CLs) and EDSS within the RRMS group. The reason for the lack of correlation in the SPMS group may be likely found in the low number of patients included in the study and in the lower number of QSM-hyperintense CLs; this possibility is in line with the neuropathologic observation of the higher frequency of chronic active lesions in the examined postmortem SPMS brains. Nevertheless, the correlation with disability has not been found with hypointense or isointense CLs because of the possible detrimental role of chronic activated microglia. ${ }^{57}$ Further longitudinal studies on larger sample sizes may clarify this interesting clinical point.

\section{CONCLUSIONS}

Our study revealed that CLs in MS are heterogeneous during the entire course of the disease, not only among patients but also within the same patient and, sometimes, within the same lesion. These results corroborate the hypothesis of substantial clinical and immunopathologic heterogeneous patterns of MS inflammation/demyelination during the disease course. Moreover, the highest frequency of QSM-hyperintense CLs in RRMS and their correlation with EDSS in this group of patients seem to suggest a key role of activated microglia/macrophages in the early and acute phases of the disease. The combined use of QSM and DIR could be a useful tool to monitor the disease evolution and to identify those patients with higher rates of inflammatory cortical demyelination and associated neurodegeneration.

Disclosures: Roberta Magliozzi-RELATED: Grant: Italian Ministry of Health, Comments: Young Investigator Grant GR-2010-2313255*. Richard Reynolds-RELATED: Grant: UK Multiple Sclerosis Society, Comments: funded the work performed in the UK at Imperial College. ${ }^{*}$ Massimiliano Calabrese_UNRELATED: Board Membership: Biogen Idec, Teva Pharmaceutical Industries; Consultancy: Genzyme; Travel/Accommodations/Meeting Expenses Unrelated to Activities Listed: Biogen Idec, Genzyme, Teva Pharmaceutical Industries, Novartis. *Money paid to the institution.

\section{REFERENCES}

1. Calabrese M, Magliozzi R, Ciccarelli O, et al. Exploring the origins of grey matter damage in multiple sclerosis. Nat Rev Neurosci 2015; 16 : 147-58 CrossRef Medline

2. Lassmann H. Multiple sclerosis: lessons from molecular neuropathology. Exp Neurol 2014;262(pt A):2-7 CrossRef Medline

3. Magliozzi R, Howell O, Vora A, et al. Meningeal B-cell follicles in secondary progressive multiple sclerosis associate with early onset of disease and severe cortical pathology. Brain 2007;130:1089-104 Medline

4. Calabrese M, Atzori M, Bernardi V, et al. Cortical atrophy is relevant in multiple sclerosis at clinical onset. J Neurol 2007;254:1212-20 Medline

5. Calabrese M, Poretto V, Favaretto A, et al. Cortical lesion load associates with progression of disability in multiple sclerosis. Brain 2012;135:2952-61 CrossRef Medline

6. Brink BP, Veerhuis R, Breij EC, et al. The pathology of multiple sclerosis is location-dependent: no significant complement activation is detected in purely cortical lesions. J Neuropathol Exp Neurol 2005;64:147-55 CrossRef Medline

7. van Horssen J, Brink BP, de Vries HE, et al. The blood-brain barrier 
in cortical multiple sclerosis lesions. J Neuropathol Exp Neurol 2007; 66:321-28 CrossRef Medline

8. Peterson JW, Bö L, Mörk S, et al. Transected neurites, apoptotic neurons, and reduced inflammation in cortical multiple sclerosis lesions. Ann Neurol 2001;50:389-400 CrossRef Medline

9. Lucchinetti CF, Popescu BFG, Bunyan RF, et al. Inflammatory cortical demyelination in early multiple sclerosis. N Engl J Med 2011; 365:2188-97 CrossRef Medline

10. Pitt D, Boster A, Pei W, et al. Imaging cortical lesions in multiple sclerosis with ultra-high-field magnetic resonance imaging. Arch Neurol 2010;67:812-18 CrossRef Medline

11. Li X, Harrison DM, Liu H, et al. Magnetic susceptibility contrast variations in multiple sclerosis lesions. J Magn Reson Imaging 2016; 43:463-73 CrossRef Medline

12. Kooi EJ, Strijbis EM, van der Valk P, et al. Heterogeneity of cortical lesions in multiple sclerosis: clinical and pathologic implications. Neurology 2012;79:1369-76 CrossRef Medline

13. Yao B, Hametner S, van Gelderen $P$, et al. 7 Tesla magnetic resonance imaging to detect cortical pathology in multiple sclerosis. PLoS One 2014;9:e108863 CrossRef Medline

14. Deistung A, Rauscher A, Sedlacik J, et al. Susceptibility weighted imaging at ultra high magnetic field strengths: theoretical considerations and experimental results. Magn Reson Med 2008;60: 1155-68 CrossRef Medline

15. Haacke EM, Xu Y, Cheng YC, et al. Susceptibility weighted imaging (SWI). Magn Reson Med 2004;52:612-18 CrossRef Medline

16. Li TQ, van Gelderen $\mathrm{P}$, Merkle $\mathrm{H}$, et al. Extensive heterogeneity in white matter intensity in high-resolution T2*-weighted MRI of the human brain at 7.0 T. Neuroimage 2006;32:1032-40 CrossRef Medline

17. Hammond KE, Metcalf M, Carvajal L, et al. Quantitative in vivo magnetic resonance imaging of multiple sclerosis at 7 Tesla with sensitivity to iron. Ann Neurol 2008;64:707-13 CrossRef Medline

18. Hagemeier J, Weinstock-Guttman B, Bergsland N, et al. Iron deposition on SWI-filtered phase in the subcortical deep gray matter of patients with clinically isolated syndrome may precede structurespecific atrophy. AJNR Am J Neuroradiol 2012;33:1596-601 CrossRef Medline

19. de Rochefort L, Liu T, Kressler B, et al. Quantitative susceptibility map reconstruction from MR phase data using Bayesian regularization: validation and application to brain imaging. Magn Reson Med 2010;63:194-206 CrossRef Medline

20. Langkammer C, Liu T, Khalil M, et al. Quantitative susceptibility mapping in multiple sclerosis. Radiology 2013;267:551-59 CrossRef Medline

21. Chen W, Gauthier SA, Gupta A, et al. Quantitative susceptibility mapping of multiple sclerosis lesions at various ages. Radiology 2014;271:183-92 CrossRef Medline

22. Bian $\mathrm{W}$, Tranvinh $\mathrm{E}$, Tourdias $\mathrm{T}$, et al. In vivo $7 \mathrm{~T}$ MR quantitative susceptibility mapping reveals opposite susceptibility contrast between cortical and white matter lesions in multiple sclerosis. $A J N R$ Am J Neuroradiol 2016 Jun 9. [Epub ahead of print] CrossRef Medline

23. Howell OW, Reeves CA, Nicholas R, et al. Meningeal inflammation is widespread and linked to cortical pathology in multiple sclerosis. Brain 2011;134:2755-71 CrossRef Medline

24. Reynolds R, Roncaroli F, Nicholas R, et al. The neuropathological basis of clinical progression in multiple sclerosis. Acta Neuropathol 2011;122:155-70 CrossRef Medline

25. Polman CH, Reingold SC, Banwell B, et al. Diagnostic criteria for multiple sclerosis: 2010 revisions to the McDonald criteria. Ann Neurol 2011;69:292-302 CrossRef Medline

26. Kurtzke JF. Rating neurologic impairment in multiple sclerosis: an Expanded Disability Status Scale (EDSS). Neurology 1983;33: 1444-52 CrossRef Medline

27. Langkammer C, Bredies K, Poser BA, et al. Fast quantitative susceptibility mapping using 3D EPI and total generalized variation. $\mathrm{Neu}$ roimage 2015;111:622-30 CrossRef Medline

28. Geurts JJ, Roosendaal SD, Calabrese M, et al; MAGNIMS Study
Group. Consensus recommendations for MS cortical lesion scoring using double inversion recovery MRI. Neurology 2011;76:418-24 CrossRef Medline

29. Avants BB, Tustison NJ, Wu J, et al. An open source multivariate framework for $\mathbf{n}$-tissue segmentation with evaluation on public data. Neuroinformatics 2011;9:381-400 CrossRef Medline

30. Calabrese M, Castellaro M, Bertoldo A, et al. Epilepsy in multiple sclerosis: the role of temporal lobe damage. Mult Scler 2016 Jun 3. [Epub ahead of print] CrossRef Medline

31. Zhang Y, Gauthier SA, Gupta A, et al. Longitudinal change in magnetic susceptibility of new enhanced multiple sclerosis (MS) lesions measured on serial quantitative susceptibility mapping (QSM). J Magn Reson Imaging 2016;44:426-32 CrossRef Medline

32. Zhang Y, Gauthier SA, Gupta A, et al. Quantitative susceptibility mapping and $\mathrm{R} 2^{\star}$ measured changes during white matter lesion development in multiple sclerosis: myelin breakdown, myelin debris degradation and removal, and iron accumulation. AJNR Am J Neuroradiol 2016;37:1629-35 CrossRef Medline

33. Zhang Y, Gauthier SA, Gupta A, et al. Magnetic susceptibility from quantitative susceptibility mapping can differentiate new enhancing from nonenhancing multiple sclerosis lesions without gadolinium injection. AJNR Am J Neuroradiol 2016 Jun 30. [Epub ahead of print] CrossRef Medline

34. Kidd D, Barkhof F, McConnell R, et al. Cortical lesions in multiple sclerosis. Brain 1999;122 (pt 1):17-26 CrossRef Medline

35. Peruzzo D, Castellaro M, Calabrese M, et al. Heterogeneity of cortical lesions in multiple sclerosis: an MRI perfusion study. J Cereb Blood Flow Metab 2013;33:457-63 CrossRef Medline

36. Seewann A, Vrenken H, Kooi E-J, et al. Imaging the tip of the iceberg: visualization of cortical lesions in multiple sclerosis. Mult Scler J 2011;17:1202-10 CrossRef Medline

37. Sethi V, Yousry TA, Muhlert N, et al. Improved detection of cortical MS lesions with phase-sensitive inversion recovery MRI. J Neurol Neurosurg Psychiatry 2012;83:877-82 CrossRef Medline

38. Wharton S, Bowtell R. Fiber orientation-dependent white matter contrast in gradient echo MRI. Proc Natl Acad Sci U S A 2012;109: 18559-64 CrossRef Medline

39. Bagnato F, Hametner S, Yao B, et al. Tracking iron in multiple sclerosis: a combined imaging and histopathological study at $7 \mathrm{Te}-$ sla. Brain 2011;134(pt 12):3602-15 CrossRef Medline

40. Yao B, Bagnato F, Matsuura E, et al. Chronic multiple sclerosis lesions: characterization with high-field-strength MR imaging. $R a$ diology 2012;262:206-15 CrossRef Medline

41. Langkammer C, Krebs N, Goessler W, et al. Quantitative MR imaging of brain iron: a postmortem validation study. Radiology 2010; 257:455-62 CrossRef Medline

42. Lee J, Shmueli K, Kang BT, et al. The contribution of myelin to magnetic susceptibility-weighted contrasts in high-field MRI of the brain. Neuroimage 2012;59:3967-75 CrossRef Medline

43. Li W, Wu B, Liu C. Quantitative susceptibility mapping of human brain reflects spatial variation in tissue composition. Neuroimage 2011;55:1645-56 CrossRef Medline

44. Schweser F, Deistung A, Lehr BW, et al. Quantitative imaging of intrinsic magnetic tissue properties using MRI signal phase: an approach to in vivo brain iron metabolism? Neuroimage 2011;54: 2789-807 CrossRef Medline

45. Langkammer C, Schweser F, Krebs N, et al. Quantitative susceptibility mapping (QSM) as a means to measure brain iron? A post mortem validation study. Neuroimage 2012;62:1593-99 CrossRef Medline

46. Li W, Wu B, Avram AV, et al. Magnetic susceptibility anisotropy of human brain in vivo and its molecular underpinnings. Neuroimage 2012;59:2088-97 CrossRef Medline

47. Kutzelnigg A, Lucchinetti CF, Stadelmann C, et al. Cortical demyelination and diffuse white matter injury in multiple sclerosis. Brain 2005;128:2705-12 CrossRef Medline

48. Craelius W, Migdal MW, Luessenhop CP, et al. Iron deposits sur- 
rounding multiple sclerosis plaques. Arch Pathol Lab Med 1982;106: 397-99 Medline

49. Bagnato F, Ikonomidou VN, van Gelderen P, et al. Lesions by tissue specific imaging characterize multiple sclerosis patients with more advanced disease. Mult Scler 2011;17:1424-31 CrossRef Medline

50. Kakeda S, Futatsuya K, Ide S, et al. Improved detection of cortical gray matter involvement in multiple sclerosis with quantitative susceptibility mapping. Acad Radiol 2015;22:1427-32 CrossRef Medline

51. Stüber C, Pitt D, Wang Y. Iron in multiple sclerosis and its noninvasive imaging with quantitative susceptibility mapping. Int J Mol Sci 2016;17(1) CrossRef Medline

52. Hametner S, Wimmer I, Haider L, et al. Iron and neurodegeneration in the multiple sclerosis brain. Ann Neurol 2013;74:848-61 CrossRef Medline

53. Paling D, Tozer D, Wheeler-Kingshott $\mathrm{C}$, et al. Reduced R2' in mul- tiple sclerosis normal appearing white matter and lesions may reflect decreased myelin and iron content. J Neurol Neurosurg Psychiatry 2012;83:785-92 CrossRef Medline

54. Khalil M, Langkammer C, Pichler A, et al. Dynamics of brain iron levels in multiple sclerosis: a longitudinal 3T MRI study. Neurology 2015;84:2396-402 CrossRef Medline

55. Schenck JF, Zimmerman EA. High-field magnetic resonance imaging of brain iron: birth of a biomarker? NMR Biomed 2004;17: 433-45 CrossRef Medline

56. Calabrese M, Reynolds R, Magliozzi R, et al. Regional distribution and evolution of gray matter damage in different populations of multiple sclerosis patients. PLoS One 2015;10:e0135428 CrossRef Medline

57. Trapp BD, Wujek JR, Criste GA, et al. Evidence for synaptic stripping by cortical microglia. Glia 2007;55:360-68 CrossRef Medline 